\title{
New types of hesitant fuzzy sets on UP-algebras
}

\author{
Phakawat Mosrijai, Akarachai Satirad, Aiyared Iampan*
}

\begin{abstract}
The concepts of sup-hesitant fuzzy UP-subalgebras, suphesitant fuzzy UP-filters, sup-hesitant fuzzy UP-ideals, and sup-hesitant fuzzy strongly UP-ideals are introduced, proved some results and discussed the generalizations of these concepts. Furthermore, we discuss the relations between sup-hesitant fuzzy UP-subalgebras (resp., suphesitant fuzzy UP-filters, sup-hesitant fuzzy UP-ideals, and sup-hesitant fuzzy strongly UP-ideals) and their level subsets.
\end{abstract}

\section{INTRODUCTION}

A hesitant fuzzy set is a function from a reference set to a power set of the unit interval. The concept of a hesitant fuzzy set was first introduced by Torra and Narukawa [15] and Torra [14] in 2009-2010. The hesitant fuzzy set theories developed by Torra and Narukawa, and others have found many applications in the domain of mathematics and elsewhere.

After the introduction of the concept of hesitant fuzzy sets by Torra and Narukawa [15, 14], several researches were conducted on the generalizations of the concept of hesitant fuzzy sets and application to many logical algebras such as: In 2012, Zhu et al. [17] introduced the concept of dual hesitant fuzzy sets, which is a new extension of fuzzy sets. In 2014, Jun et al. [5] introduced the concepts of hesitant fuzzy soft subalgebras and (closed) hesitant fuzzy soft ideals in BCK/BCI-algebras. Jun and Song [6] introduced the concepts of (Boolean, prime, ultra, good) hesitant fuzzy filters and hesitant fuzzy MVfilters of MTL-algebras. In 2015, Jun and Song [7] introduced the concepts of hesitant fuzzy prefilters (resp., filters) and positive implicative hesitant fuzzy prefilters (resp., filters) of EQ-algebras. In 2016, Jun and Ahn [4] introduced the concepts of hesitant fuzzy subalgebras and hesitant fuzzy ideals of BCK/BCI-algebras. Rezaei and Borumand Saeid [11] introduced

2000 Mathematics Subject Classification. Primary: 03G25; Secondary: 08A72.

Key words and phrases. UP-algebra, sup-hesitant fuzzy UP-subalgebra, sup-hesitant fuzzy UP-filter, sup-hesitant fuzzy UP-ideal, and sup-hesitant fuzzy strongly UP-ideal.

Full paper. Received 14 May 2018, revised 5 June 2018, accepted 13 July 2018, available online 15 December 2018.

This work was financially supported by the University of Phayao.

${ }^{*}$ Corresponding author. 
the concept of hesitant fuzzy (implicative) filters on BE-algebras. In 2017, Mosrijai et al. [9] introduced the concept of hesitant fuzzy sets on UPalgebras. Satirad et al. [12] explicitly described the relationships among (prime, weakly prime) hesitant fuzzy UP-subalgebras (resp., hesitant fuzzy UP-filters, hesitant fuzzy UP-ideals and hesitant fuzzy strongly UP-ideals) and their level subsets on UP-algebras. In 2018, Mosrijai et al. [10] defined the partial constant hesitant fuzzy sets on UP-algebras. Mosrijai and Iampan [8] introduced the concepts of anti-hesitant fuzzy UP-subalgebras of UPalgebras, anti-hesitant fuzzy UP-filters, anti-hesitant fuzzy UP-ideals, and anti-hesitant fuzzy strongly UP-ideals of UP-algebras.

In present paper, the concepts of sup-hesitant fuzzy UP-subalgebras, suphesitant fuzzy UP-filters, sup-hesitant fuzzy UP-ideals, and sup-hesitant fuzzy strongly UP-ideals are introduced, proved some results and discussed the generalizations of these notions. Furthermore, we discuss the relations between sup-hesitant fuzzy UP-subalgebras (resp., sup-hesitant fuzzy UPfilters, sup-hesitant fuzzy UP-ideals, and sup-hesitant fuzzy strongly UPideals) and their level subsets.

\section{Preliminaries}

Before we start our study, we recall some definitions and facts about UPalgebras.

An algebra $A=(A, \cdot, 0)$ of type $(2,0)$ is called a UP-algebra [2] where $A$ is a nonempty set, $\cdot$ is a binary operation on $A$, and 0 is a fixed element of $A$ if it satisfies the following assertions:

(UP-1): (for all $x, y, z \in A)((y \cdot z) \cdot((x \cdot y) \cdot(x \cdot z))=0)$,

(UP-2): (for all $x \in A)(0 \cdot x=x)$,

(UP-3): (for all $x \in A)(x \cdot 0=0)$, and

(UP-4): (for all $x, y \in A)(x \cdot y=0, y \cdot x=0 \Rightarrow x=y)$.

From [2], we know that a UP-algebra is a generalization of the concept of a KU-algebra.

In a UP-algebra $A=(A, \cdot, 0)$, the following assertions are valid (see $[2,3])$.

(for all $x \in A)(x \cdot x=0)$,

(for all $x, y, z \in A)(x \cdot y=0, y \cdot z=0 \Rightarrow x \cdot z=0$ ),

(for all $x, y, z \in A)(x \cdot y=0 \Rightarrow(z \cdot x) \cdot(z \cdot y)=0)$,

(for all $x, y, z \in A)(x \cdot y=0 \Rightarrow(y \cdot z) \cdot(x \cdot z)=0)$,

(for all $x, y \in A)(x \cdot(y \cdot x)=0)$,

(for all $x, y \in A)((y \cdot x) \cdot x=0 \Leftrightarrow x=y \cdot x)$,

(for all $x, y \in A)(x \cdot(y \cdot y)=0)$,

$($ for all $x, y, z, a \in A)((x \cdot(y \cdot z)) \cdot(x \cdot((a \cdot y) \cdot(a \cdot z)))=0)$, 
(for all $x, y, z, a \in A)((((a \cdot x) \cdot(a \cdot y)) \cdot z) \cdot((x \cdot y) \cdot z)=0)$, (for all $x, y, z \in A)(((x \cdot y) \cdot z) \cdot(y \cdot z)=0)$, (for all $x, y, z \in A)(x \cdot y=0 \Rightarrow x \cdot(z \cdot y)=0)$, (for all $x, y, z \in A)(((x \cdot y) \cdot z) \cdot(x \cdot(y \cdot z))=0)$, and (for all $x, y, z, a \in A)(((x \cdot y) \cdot z) \cdot(y \cdot(a \cdot z))=0)$.

We assume from now on that $A$ will always denote a $\operatorname{UP}$-algebra $(A, \cdot, 0)$.

Definition 2.1 ([2]). A subset $S$ of $A$ is called a UP-subalgebra of $A$ if the constant 0 of $A$ is in $S$, and $(S, \cdot, 0)$ itself forms a UP-algebra.

Iampan [2] proved the useful criteria that a nonempty subset $S$ of $A$ is a UP-subalgebra of $A$ if and only if $S$ is closed under the - multiplication on A.

Definition $2.2([2,13,1])$. A subset $S$ of $A$ is called

(1) a UP-ideal of $A$ if

(i) the constant 0 of $A$ is in $S$, and

(ii) (for all $x, y, z \in A)(x \cdot(y \cdot z) \in S, y \in S \Rightarrow x \cdot z \in S$ ).

(2) a UP-filter of $A$ if

(i) the constant 0 of $A$ is in $S$, and

(ii) (for all $x, y \in A)(x \cdot y \in S, x \in S \Rightarrow y \in S$ ).

(3) a strongly UP-ideal of $A$ if

(i) the constant 0 of $A$ is in $S$, and

(ii) (for all $x, y, z \in A)((z \cdot y) \cdot(z \cdot x) \in S, y \in S \Rightarrow x \in S$ ).

Guntasow et al. [1] proved that a UP-subalgebra is a generalization of the concept of a UP-filter, a UP-filter is a generalization of the concept of a UP-ideal, and a UP-ideal is a generalization of the concept of a strongly UP-ideal. Moreover, they also proved that a UP-algebra $A$ is the only one strongly UP-ideal of itself.

A fuzzy set [16] in a nonempty set $X$ (or a fuzzy subset of $X$ ) is an arbitrary function from the set $X$ into $[0,1]$ where $[0,1]$ is the unit segment of the real line.

Somjanta et al. [13] and Guntasow et al. [1] introduced the concept of fuzzy UP-subalgebras (resp., fuzzy UP-filters, fuzzy UP-ideals, fuzzy strongly UP-ideals) of UP-algebras as follows:

Definition 2.3. A fuzzy set $f$ in $A$ is called

(1) a fuzzy UP-subalgebra of $A$ if for any (for all $x, y \in A)(f(x \cdot y) \geq$ $\min \{f(x), f(y)\})$.

(2) a fuzzy UP-filter of $A$ if

(i) (for all $x \in A)(f(0) \geq f(x)$ ), and

(ii) (for all $x, y \in A)(f(y) \geq \min \{f(x \cdot y), f(x)\}$ ).

(3) a fuzzy UP-ideal of $A$ if 
(i) (for all $x \in A)(f(0) \geq f(x)$ ), and

(ii) (for all $x, y, z \in A)(f(x \cdot z) \geq \min \{f(x \cdot(y \cdot z)), f(y)\})$.

(4) a fuzzy strongly UP-ideal of $A$ if

(i) (for all $x \in A)(f(0) \geq f(x))$, and

(ii) (for all $x, y, z \in A)(f(x) \geq \min \{f((z \cdot y) \cdot(z \cdot x)), f(y)\})$.

Guntasow et al. [1] also proved that a fuzzy UP-subalgebra is a generalization of the concept of a fuzzy UP-filter, a fuzzy UP-filter is a generalization of the concept of a fuzzy UP-ideal, and a fuzzy UP-ideal is a generalization of the concept of a fuzzy strongly UP-ideal. Moreover, they also proved that a fuzzy strongly UP-ideal and a constant function coincide.

Let $X$ be a reference set. A hesitant fuzzy set [14] on $X$ is defined in term of a function $\mathrm{h}$ that when applied to $X$ return a subset of $[0,1]$, that is, $\mathrm{h}: X \rightarrow \mathcal{P}([0,1])$. Let $\mathrm{h}$ be a hesitant fuzzy set on $X$. The hesitant fuzzy set $\overline{\mathrm{h}}$ defined by $\overline{\mathrm{h}}(x)=[0,1]-\mathrm{h}(x)$ for all $x \in X$ is said to be the complement [9] of $\mathrm{h}$ on $X$. The hesitant fuzzy set $\mathrm{h}^{*}$ defined by $\mathrm{h}^{*}(x)=$ $\{1-\operatorname{suph}(x)\}$ for all $x \in X$ is said to be the supremum complement of $\mathrm{h}$ on $X$. Then $\operatorname{suph}^{*}(x)=1-\operatorname{suph}(x)$ for all $x \in X$. We observe that $\left(\mathrm{h}^{*}\right)^{*}(x)=\{\operatorname{suph}(x)\}$ for all $x \in X$, and then $\sup \left(\mathrm{h}^{*}\right)^{*}(x)=\operatorname{suph}(x)$ for all $x \in X$.

Definition 2.4. A hesitant fuzzy set $\mathrm{h}$ on $A$ is called

(1) a sup-hesitant fuzzy UP-subalgebra of $A$ if (for all $x, y \in A)(\operatorname{suph}(x$. $y) \geq \min \{\operatorname{suph}(x), \operatorname{suph}(y)\})$.

(2) a sup-hesitant fuzzy UP-filter of $A$ if

(i) (for all $x \in A)(\operatorname{suph}(0) \geq \operatorname{suph}(x))$, and

(ii) (for all $x, y \in A)(\operatorname{suph}(y) \geq \min \{\operatorname{suph}(x \cdot y), \operatorname{suph}(x)\})$.

(3) a sup-hesitant fuzzy UP-ideal of $A$ if

(i) (for all $x \in A)(\operatorname{suph}(0) \geq \operatorname{suph}(x))$, and

(ii) (for all $x, y, z \in A)(\operatorname{suph}(x \cdot z) \geq \min \{\operatorname{suph}(x \cdot(y \cdot z)), \operatorname{suph}(y)\})$.

(4) a sup-hesitant fuzzy strongly UP-ideal of $A$ if

(i) (for all $x \in A)(\operatorname{suph}(0) \geq \operatorname{suph}(x))$, and

(ii) (for all $x, y, z \in A)(\operatorname{suph}(x) \geq \min \{\operatorname{suph}((z \cdot y) \cdot(z \cdot x)), \operatorname{suph}(y)\})$.

If $\mathrm{h}$ is a sup-hesitant fuzzy UP-subalgebra of $A$, then it follows from (2.1) that $\operatorname{suph}(0)=\operatorname{suph}(x \cdot x) \geq \min \{\operatorname{suph}(x), \operatorname{suph}(x)\}=\operatorname{suph}(x)$ for all $x \in A$.

If $f$ is a fuzzy set in $A$, we define a hesitant fuzzy set $\mathrm{h}_{f}$ on $A$ by $\mathrm{h}_{f}(x)=$ $\{f(x)\}$ for all $x \in A$, and then $\operatorname{suph}_{f}(x)=f(x)$ for all $x \in A$. We call $\mathrm{h}_{f}$ the hesitant fuzzy set induced by $f$. We can easily show that if $f$ is a fuzzy UPsubalgebra (resp., fuzzy UP-filter, fuzzy UP-ideal, fuzzy strongly UP-ideal) of $A$, then $\mathrm{h}_{f}$ is a sup-hesitant fuzzy UP-subalgebra (resp., sup-hesitant fuzzy UP-filter, sup-hesitant fuzzy UP-ideal, sup-hesitant fuzzy strongly UP-ideal) of $A$. 
If $\mathrm{h}$ is a hesitant fuzzy set on $A$, we define a fuzzy set $f_{\mathrm{h}}$ in $A$ by $f_{\mathrm{h}}(x)=$ $\operatorname{suph}(x)$ for all $x \in A$. We call $f_{\mathrm{h}}$ the fuzzy set induced by h. We can easily show that if $\mathrm{h}$ is a sup-hesitant fuzzy UP-subalgebra (resp., sup-hesitant fuzzy UP-filter, sup-hesitant fuzzy UP-ideal, sup-hesitant fuzzy strongly UPideal) of $A$, then $f_{\mathrm{h}}$ is a fuzzy UP-subalgebra (resp., fuzzy UP-filter, fuzzy UP-ideal, fuzzy strongly UP-ideal) of $A$.

By Definition 2.4, we will discuss the generalizations of these concepts.

Theorem 2.1. Every sup-hesitant fuzzy UP-filter of $A$ is a sup-hesitant fuzzy UP-subalgebra.

Proof. Given that $\mathrm{h}$ is a sup-hesitant fuzzy UP-filter of $A$. Then for all $x, y \in A$,

$$
\begin{aligned}
\operatorname{suph}(x \cdot y) & \geq \min \{\operatorname{suph}(y \cdot(x \cdot y)), \operatorname{suph}(y)\} \\
& =\min \{\operatorname{suph}(0), \operatorname{suph}(y)\} \\
& =\operatorname{suph}(y) \\
& \geq \min \{\operatorname{suph}(x), \operatorname{suph}(y)\}
\end{aligned}
$$

Hence, $\mathrm{h}$ is a sup-hesitant fuzzy UP-subalgebra of $A$.

Example 2.1. Consider a UP-algebra $A=\{0,1,2,3\}$ with the below Cayley table:

\begin{tabular}{c|cccc}
$\cdot$ & 0 & 1 & 2 & 3 \\
\hline 0 & 0 & 1 & 2 & 3 \\
1 & 0 & 0 & 1 & 2 \\
2 & 0 & 0 & 0 & 1 \\
3 & 0 & 0 & 0 & 0
\end{tabular}

We define a hesitant fuzzy set $\mathrm{h}$ on $A$ as follows:

$$
\mathrm{h}(0)=[0,1], \mathrm{h}(1)=[0.5,0.6], \mathrm{h}(2)=(0.1,0.4) \text {, and } \mathrm{h}(3)=[0,0.1) .
$$

Then $\mathrm{h}$ is a sup-hesitant fuzzy UP-subalgebra of $A$. Since $\operatorname{suph}(2)=0.4 \ngtr$ $0.6=\min \{\operatorname{suph}(1 \cdot 2), \operatorname{suph}(1)\}$, we have $\mathrm{h}$ is not a sup-hesitant fuzzy UPfilter of $A$.

Theorem 2.2. Every sup-hesitant fuzzy UP-ideal of $A$ is a sup-hesitant fuzzy UP-filter.

Proof. Given that $\mathrm{h}$ is a sup-hesitant fuzzy UP-ideal of $A$. Then for all $x, y \in A, \operatorname{suph}(0) \geq \operatorname{suph}(x)$, and

$$
\begin{aligned}
((\mathrm{UP}-2)) \quad \operatorname{suph}(y) & =\operatorname{suph}(0 \cdot y) \\
& \geq \min \{\operatorname{suph}(0 \cdot(x \cdot y)), \operatorname{suph}(x)\} \\
((\mathrm{UP}-2)) & =\min \{\operatorname{suph}(x \cdot y), \operatorname{suph}(x)\} .
\end{aligned}
$$

Hence, $\mathrm{h}$ is a sup-hesitant fuzzy UP-filter of $A$. 
Example 2.2. Consider a UP-algebra $A=\{0,1,2,3\}$ with the below Cayley table:

\begin{tabular}{c|cccc}
$\cdot$ & 0 & 1 & 2 & 3 \\
\hline 0 & 0 & 1 & 2 & 3 \\
1 & 0 & 0 & 2 & 2 \\
2 & 0 & 1 & 0 & 2 \\
3 & 0 & 1 & 0 & 0
\end{tabular}

We define a hesitant fuzzy set $\mathrm{h}$ on $A$ as follows:

$$
\mathrm{h}(0)=[0.5,1), \mathrm{h}(1)=(0,0.2), \mathrm{h}(2)=[0,0.1] \text {, and } \mathrm{h}(3)=(0,0.1] .
$$

Then $\mathrm{h}$ is a sup-hesitant fuzzy UP-filter of $A$. Since $\operatorname{suph}(2 \cdot 3)=0.1 \nsupseteq$ $0.2=\min \{\operatorname{suph}(2 \cdot(1 \cdot 3)), \operatorname{suph}(1)\}$, we have $\mathrm{h}$ is not a sup-hesitant fuzzy UP-ideal of $A$.

Theorem 2.3. Every sup-hesitant fuzzy strongly UP-ideal of $A$ is a suphesitant fuzzy UP-ideal.

Proof. Given that $\mathrm{h}$ is a sup-hesitant fuzzy strongly UP-ideal of $A$. Then for all $x, y, z \in A, \operatorname{suph}(0) \geq \operatorname{suph}(x)$, and

$$
\begin{aligned}
\operatorname{suph}(x \cdot z) & \geq \min \{\operatorname{suph}((z \cdot y) \cdot(z \cdot(x \cdot z))), \operatorname{suph}(y)\} \\
& =\min \{\operatorname{suph}((z \cdot y) \cdot 0), \operatorname{suph}(y)\} \\
& =\min \{\operatorname{suph}(0), \operatorname{suph}(y)\} \\
& =\operatorname{suph}(y) \\
& \geq \min \{\operatorname{suph}(x \cdot(y \cdot z)), \operatorname{suph}(y)\} .
\end{aligned}
$$

Hence, $\mathrm{h}$ is a sup-hesitant fuzzy UP-ideal of $A$.

Example 2.3. Consider a UP-algebra $A=\{0,1,2,3\}$ with the below Cayley table:

\begin{tabular}{c|cccc}
$\cdot$ & 0 & 1 & 2 & 3 \\
\hline 0 & 0 & 1 & 2 & 3 \\
1 & 0 & 0 & 2 & 3 \\
2 & 0 & 1 & 0 & 3 \\
3 & 0 & 1 & 2 & 0
\end{tabular}

We define a hesitant fuzzy set $\mathrm{h}$ on $A$ as follows:

$$
\mathrm{h}(0)=\{0.6\}, \mathrm{h}(1)=\{0.4\}, \mathrm{h}(2)=(0,0.3) \text {, and } \mathrm{h}(3)=(0,0.2] .
$$

Then $\mathrm{h}$ is a sup-hesitant fuzzy UP-ideal of $A$. Since $\operatorname{suph}(1)=0.4 \nsupseteq 0.6=$ $\min \{\operatorname{suph}((1 \cdot 0) \cdot(1 \cdot 1)), \operatorname{suph}(0)\}$, we have $\mathrm{h}$ is not a sup-hesitant fuzzy strongly UP-ideal of $A$.

By Theorems 2.1, 2.2, and 2.3 and Examples 2.1, 2.2, and 2.3, we have that a sup-hesitant fuzzy UP-subalgebra is a generalization of the concept of a sup-hesitant fuzzy UP-filter, a sup-hesitant fuzzy UP-filter is a generalization of the concept of a sup-hesitant fuzzy UP-ideal, and a sup-hesitant fuzzy UP-ideal is a generalization of the concept of a sup-hesitant fuzzy strongly UP-ideal. 
Theorem 2.4. A hesitant fuzzy set h on $A$ is a sup-hesitant fuzzy strongly UP-ideal of $A$ if and only if the supremum of all images of $\mathrm{h}$ is equal.

Proof. Given that $\mathrm{h}$ is a sup-hesitant fuzzy strongly UP-ideal of $A$. Then $\operatorname{suph}(0) \geq \operatorname{suph}(x)$ and $\operatorname{suph}(x) \geq \min \{\operatorname{suph}((z \cdot y) \cdot(z \cdot x)), \operatorname{suph}(y)\}$ for all $x, y, z \in A$. For any $x \in A$, we choose $z=x$ and $y=0$. Then

$((\mathrm{UP}-3)$ and $(2.1))$

$$
\begin{aligned}
\operatorname{suph}(x) & \geq \min \{\operatorname{suph}((x \cdot 0) \cdot(x \cdot x)), \operatorname{suph}(0)\} \\
& =\min \{\operatorname{suph}(0 \cdot 0), \operatorname{suph}(0)\} \\
& =\min \{\operatorname{suph}(0), \operatorname{suph}(0)\} \\
& =\operatorname{suph}(0) \\
& \geq \operatorname{suph}(x),
\end{aligned}
$$

so $\operatorname{suph}(x)=\operatorname{suph}(0)$ for all $x \in A$.

Conversely, given that the supremum of all images of $\mathrm{h}$ is equal. Then, for all $x \in A, \operatorname{suph}(x)=\operatorname{suph}(0)$. Obviously, $\operatorname{suph}(0) \geq \operatorname{suph}(x)$ and $\operatorname{suph}(x) \geq \min \{\operatorname{suph}((z \cdot y) \cdot(z \cdot x)), \operatorname{suph}(y)\}$ for all $x, y, z \in A$. Hence, $\mathrm{h}$ is a sup-hesitant fuzzy strongly UP-ideal of $A$.

\section{Characterizations of $t$-Level Subsets}

We divide this section into two parts. We study sup-upper $t$-level subsets in the first part, and sup-lower $t$-level subsets in the second part.

Definition 3.1. Let $\mathrm{h}$ be a hesitant fuzzy set on $A$. For any $t \in[0,1]$, the sets

$U_{\text {sup }}(\mathrm{h} ; t)=\{x \in A \mid \operatorname{suph}(x) \geq t\}$ and $L_{\text {sup }}(\mathrm{h} ; t)=\{x \in A \mid \operatorname{suph}(x) \leq t\}$ are called a sup-upper t-level subset and a sup-lower $t$-level subset of $\mathrm{h}$, respectively.

3.1. sup-Upper $t$-Level Subsets. In this part, we discuss the relations between sup-hesitant fuzzy UP-subalgebras (resp., sup-hesitant fuzzy UPfilters, sup-hesitant fuzzy UP-ideals, and sup-hesitant fuzzy strongly UPideals) and their sup-upper $t$-level subsets.

Theorem 3.1. A hesitant fuzzy set $\mathrm{h}$ on $A$ is a sup-hesitant fuzzy UPsubalgebra of $A$ if and only if for all $t \in[0,1]$, a nonempty subset $U_{\text {sup }}(\mathrm{h} ; t)$ of $A$ is a UP-subalgebra of $A$.

Proof. Given that h is a sup-hesitant fuzzy UP-subalgebra of $A$. Let $t \in[0,1]$ be such that $U_{\text {sup }}(\mathrm{h} ; t) \neq \emptyset$ and let $x, y \in U_{\text {sup }}(\mathrm{h} ; t)$. Then $\operatorname{suph}(x) \geq t$ and $\operatorname{suph}(y) \geq t$. Since $\mathrm{h}$ is a sup-hesitant fuzzy UP-subalgebra of $A$, we have $\operatorname{suph}(x \cdot y) \geq \min \{\operatorname{suph}(x), \operatorname{suph}(y)\} \geq t$ and so $x \cdot y \in U_{\text {sup }}(\mathrm{h} ; t)$. Therefore, $U_{\text {sup }}(\mathrm{h} ; t)$ is a UP-subalgebra of $A$.

Conversely, given that for all $t \in[0,1]$, a nonempty subset $U_{\text {sup }}(\mathrm{h} ; t)$ of $A$ is a UP-subalgebra of $A$. Let $x, y \in A$. Choose $t=\min \{\operatorname{suph}(x), \operatorname{suph}(y)\} \in$ $[0,1]$. Thus $\operatorname{suph}(x) \geq t$ and $\operatorname{suph}(y) \geq t$. Thus $x, y \in U_{\text {sup }}(\mathrm{h} ; t) \neq \emptyset$. 
By assumption, $U_{\text {sup }}(\mathrm{h} ; t)$ is a UP-subalgebra of $A$ and so $x \cdot y \in U_{\text {sup }}(\mathrm{h} ; t)$. Thus $\operatorname{suph}(x \cdot y) \geq t=\min \{\operatorname{suph}(x), \operatorname{suph}(y)\}$. Hence, $\mathrm{h}$ is a sup-hesitant fuzzy UP-subalgebra of $A$.

The proof of Theorems 3.2, 3.3, and 3.4 can be established by a similar argument to the proof of Theorem 3.1.

Theorem 3.2. A hesitant fuzzy set h on $A$ is a sup-hesitant fuzzy UP-filter of $A$ if and only if for all $t \in[0,1]$, a nonempty subset $U_{\mathrm{sup}}(\mathrm{h} ; t)$ of $A$ is a UP-filter of $A$.

Theorem 3.3. A hesitant fuzzy set $\mathrm{h}$ on $A$ is a sup-hesitant fuzzy UP-ideal of $A$ if and only if for all $t \in[0,1]$, a nonempty subset $U_{\mathrm{sup}}(\mathrm{h} ; t)$ of $A$ is a UP-ideal of $A$.

Theorem 3.4. A hesitant fuzzy set h on $A$ is a sup-hesitant fuzzy strongly $U P$-ideal of $A$ if and only if for all $t \in[0,1]$, a nonempty subset $U_{\text {sup }}(\mathrm{h} ; t)$ of $A$ is a strongly UP-ideal of $A$.

3.2. sup-Lower $t$-Level Subsets. In this part, we discuss the relations between sup-hesitant fuzzy UP-subalgebras (resp., sup-hesitant fuzzy UPfilters, sup-hesitant fuzzy UP-ideals, and sup-hesitant fuzzy strongly UPideals) and their sup-lower $t$-level subsets.

Theorem 3.5. Let $\mathrm{h}$ be a hesitant fuzzy set on $A$. Then $\mathrm{h}^{*}$ is a sup-hesitant fuzzy UP-subalgebra of $A$ if and only if for all $t \in[0,1]$, a nonempty subset $L_{\text {sup }}(\mathrm{h} ; t)$ of $A$ is a UP-subalgebra of $A$.

Proof. Given that $\mathrm{h}^{*}$ is a sup-hesitant fuzzy UP-subalgebra of $A$. Let $t \in$ $[0,1]$ be such that $L_{\text {sup }}(\mathrm{h} ; t) \neq \emptyset$ and let $x, y \in L_{\text {sup }}(\mathrm{h} ; t)$. Then $\operatorname{suph}(x) \leq t$ and $\operatorname{suph}(y) \leq t$. Since $\mathrm{h}^{*}$ is a sup-hesitant fuzzy UP-subalgebra of $A$, we have $\operatorname{suph}^{*}(x \cdot y) \geq \min \left\{\operatorname{suph}^{*}(x), \operatorname{suph}^{*}(y)\right\}$, that is, $1-\operatorname{suph}(x$. $y) \geq \min \{1-\operatorname{suph}(x), 1-\operatorname{suph}(y)\}=1-\max \{\operatorname{suph}(x), \operatorname{suph}(y)\}$. Hence, $\operatorname{suph}(x \cdot y) \leq \max \{\operatorname{suph}(x), \operatorname{suph}(y)\} \leq t$, so $x \cdot y \in L_{\text {sup }}(\mathrm{h} ; t)$. Therefore, $L_{\text {sup }}(\mathrm{h} ; t)$ is a UP-subalgebra of $A$.

Conversely, given that for all $t \in[0,1]$, a nonempty subset $L_{\text {sup }}(\mathrm{h} ; t)$ of $A$ is a UP-subalgebra of $A$. Let $x, y \in A$. Choose $t=\max \{\operatorname{suph}(x), \operatorname{suph}(y)\} \in$ $[0,1]$. Then $\operatorname{suph}(x) \leq t$ and $\operatorname{suph}(y) \leq t$. Thus $x, y \in L_{\text {sup }}(\mathrm{h} ; t) \neq \emptyset$. By assumption, we have $L_{\text {sup }}(\mathrm{h} ; t)$ is a UP-subalgebra of $A$ and so $x \cdot y \in$ $L_{\text {sup }}(\mathrm{h} ; t) . T$ Thus $\operatorname{suph}(x \cdot y) \leq t=\max \{\operatorname{suph}(x), \operatorname{suph}(y)\}$. Hence, 


$$
\begin{aligned}
\operatorname{suph}^{*}(x \cdot y) & =1-\operatorname{suph}(x \cdot y) \\
& \geq 1-\max \{\operatorname{suph}(x), \operatorname{suph}(y)\} \\
& =\min \{1-\operatorname{suph}(x), 1-\operatorname{suph}(y)\} \\
& =\min \left\{\operatorname{suph}^{*}(x), \operatorname{suph}^{*}(y)\right\} .
\end{aligned}
$$

Therefore, $\mathrm{h}^{*}$ is a sup-hesitant fuzzy UP-subalgebra of $A$.

The proof of Theorems 3.6, 3.7, and 3.8 can be established by a similar argument to the proof of Theorem 3.5.

Theorem 3.6. Let $\mathrm{h}$ be a hesitant fuzzy set on $A$. Then $\mathrm{h}^{*}$ is a suphesitant fuzzy UP-filter of $A$ if and only if for all $t \in[0,1]$, a nonempty subset $L_{\text {sup }}(\mathrm{h} ; t)$ of $A$ is a UP-filter of $A$.

Theorem 3.7. Let $\mathrm{h}$ be a hesitant fuzzy set on $A$. Then $\mathrm{h}^{*}$ is a suphesitant fuzzy UP-ideal of $A$ if and only if for all $t \in[0,1]$, a nonempty subset $L_{\mathrm{sup}}(\mathrm{h} ; t)$ of $A$ is a UP-ideal of $A$.

Theorem 3.8. Let $\mathrm{h}$ be a hesitant fuzzy set on $A$. Then $\mathrm{h}^{*}$ is a sup-hesitant fuzzy strongly UP-ideal of $A$ if and only if for all $t \in[0,1]$, a nonempty subset $L_{\text {sup }}\left(\mathrm{h}^{*} ; t\right)$ of $A$ is a strongly UP-ideal of $A$.

\section{Conclusions and Future Works}

In present paper, we have introduced the concepts of sup-hesitant fuzzy UP-sub-algebras, sup-hesitant fuzzy UP-filters, sup-hesitant fuzzy UP-ideals, and sup-hesitant fuzzy strongly UP-ideals of UP-algebras, proved their generalizations and investigated some of their important properties. Then we have the generalization diagram of hesitant fuzzy sets in the meaning of the supremum of its images in UP-algebras below.

sup-hesitant fuzzy UP-subalgebra

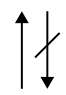

sup-hesitant fuzzy UP-filter

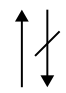

sup-hesitant fuzzy UP-ideal

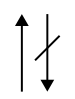

sup-hesitant fuzzy strongly UP-ideal 
In our future study of UP-algebras, the following objectives considered:

- To get more results of hesitant fuzzy sets in the meaning of the supremum of its images.

- To define anti-hesitant fuzzy sets in the meaning of the supremum of its images.

- To define operations of hesitant fuzzy sets.

- To define hesitant fuzzy soft sets.

\section{ACKNOWLEDGMENT}

The authors would also like to thank the anonymous referee for giving many helpful suggestion on the revision of present paper.

\section{REFERENCES}

[1] T. Guntasow, S. Sajak, A. Jomkham, and A. Iampan, Fuzzy translations of a fuzzy set in UP-algebras, J. Indones. Math. Soc. 23 (2017), no. 2, 1-19.

[2] A. Iampan, A new branch of the logical algebra: UP-algebras, J. Algebra Relat. Top. 5 (2017), no. $1,35-54$.

[3] A. Iampan, Introducing fully UP-semigroups, Discuss. Math., Gen. Algebra Appl., 38 (2) (2018), 297-306.

[4] Y. B. Jun and S. S. Ahn, Hesitant fuzzy set theory applied to BCI/BCK-algebras, J. Comput. Anal. Appl., 20 (4) (2016), 635-646.

[5] Y. B. Jun, S. S. Ahn, and G. Muhiuddin, Hesitant fuzzy soft subalgebra and ideal in BCI/BCK-algebras, Sci. World J. 2014 (2014), Article ID 763929, 7 pages.

[6] Y. B. Jun and S. Z. Song, Hesitant fuzzy set theory applied to filters in MTL-algebras, Honam Math. J., 36 (4) (2014), 813-830.

[7] Y. B. Jun and S. Z. Song, Hesitant fuzzy prefilters and filters of EQ-algebras, Appl. Math. Sci., 9 (11) (2015), 515-532.

[8] P. Mosrijai and A. Iampan, Anti-type of hesitant fuzzy sets on UP-algebras, Eur. J. Pure Appl. Math. 11 (4) (2018), 976-1002.

[9] P. Mosrijai, W. Kamti, A. Satirad, and A. Iampan, Hesitant fuzzy sets on UPalgebras, Konuralp J. Math., 5 (2) (2017), 268-280.

[10] P. Mosrijai, A. Satirad, and A. Iampan, Partial constant hesitant fuzzy sets on UPalgebras, J. New Theory, 22 (2018), 39-50.

[11] A. Rezaei and A. B. Saeid, Hesitant fuzzy filters in BE-algebras, Int. J. Comput. Int. Sys., 9 (1) (2016), 110-119.

[12] A. Satirad, P. Mosrijai, W. Kamti, and A. Iampan, Level subsets of a hesitant fuzzy set on UP-algebras, Ann. Fuzzy Math. Inform., 14 (3) (2017), 279-302.

[13] J. Somjanta, N. Thuekaew, P. Kumpeangkeaw, and A. Iampan, Fuzzy sets in UPalgebras, Ann. Fuzzy Math. Inform., 12 (6) (2016), 739-756.

[14] V. Torra, Hesitant fuzzy sets, Int. J. Intell. Syst., 25 (2010), 529-539. 
[15] V. Torra and Y. Narukawa, On hesitant fuzzy sets and decision, Proceedings of IEEE International Conference on Fuzzy Systems, FUZZ-IEEE 2009, Jeju Island, Korea, 20-24 August 2009, 1378-1382.

[16] L. A. Zadeh, Fuzzy sets, Inf. Cont., 8 (1965), 338-353.

[17] B. Zhu, Z. Xu, and M. Xia, Dual hesitant fuzzy sets, J. Appl. Math., 2012 (2012), Article ID: 879629, 13 pages.

Phakawat Mosrijai

Department of Mathematics

SCHOOL OF SCIENCE

University of Phayao

PhaYao 56000

THAILAND

E-mail address: phakawat.mo@gmail.com

\section{Akarachai Satirad}

Department of Mathematics

SCHOOL OF SCIENCE

University of Phayao

PHAYAO 56000

Thailand

E-mail address: akarachai.sa@gmail.com

\section{Aiyared Iampan}

Department of Mathematics

SCHOOL OF SCIENCE

University of PhayaO

PhayaO 56000

Thailand

E-mail address: aiyared.ia@up.ac.th 